\title{
Guide to the Parasites of Fishes of Canada Part V: Nematoda
}

By Hisao P. Arai and John W. Smith. Edited by Michael D.B. Burt and Donald F. McAlpine. 2016. Zootaxa 4185, Magnolia Press. 274 pages, freely available (https://biotaxa.org/Zootaxa/article/view/zootaxa.4185.1.1), PDF.

Over the last four decades, the parasites impacting Canadian fish species have been described in Volumes I-IV of the Guide to the Parasites of Fishes of Canada, with Part V: Nematoda by Hisao P. Arai and John W. Smith bringing much needed attention to the parasitic nematodes affecting Canadian fishes. Nematodes occupy nearly every habitat from soil to sea and are recognized as one of the most abundant multicellular animals on earth. Parasitic nematodes of fishes can pose a threat not only to fish health but also to the organisms (including humans) that consume these infected hosts, emphasizing the urgency to study this group of organisms.

This volume is first and foremost an identification key for the nematode parasites of Canadian fishes, pro- viding detailed information on all taxonomic levels. The taxonomy follows the broader taxonomic guides on nematodes of vertebrates while incorporating research discoveries made in recent decades. Species are described that have only just gained recognition in recent years, despite having negative economic consequences (e.g., Huffmanela canadensis, Philometra rubra, and Anguillicola crassus). The book is remarkably thorough, compiling information from an expansive reference list of over 800 citations, which is reflected in the level of detail in each key.

The keys and descriptions cover 88 species, spanning 47 genera expertly described by the authors. These keys provide a concise yet detailed description of each listed 
taxon. Each species or taxonomic rank includes a morphological description, reported hosts, host anatomical site, geographical distribution, and previous literature records. This information is coupled with over $100 \mathrm{de}-$ tailed illustrations that present nematode anatomy in a way that cannot often be appreciated within photographic images. These illustrations provide the reader with views of organisms in their entirety, and views of specific anatomy relevant to species identification. Fish parasitologists will benefit from details provided within the introduction on the procedures and protocols used to collect, examine, and identify nematodes from fish hosts. Although these components of the book will be most relevant to experts within the field, broader information on Phylum Nematoda, nematode morphology, and the geographical distributions of nematodes can be gleaned by readers lacking familiarity with this area of research. The indices by nematode and fish species are particularly valuable for readers seeking species-specif- ic information. These indices will be useful for fisheries biologists looking to understand which nematode species may be present in a target population, based on their potential hosts and geographical distributions.

The authors make it clear that morphology-based identification is still a major component of nematode taxonomy, and that molecular-based taxonomic information is lacking for most species. This book should serve as a call to action for further attention into nematode phylogenetics, as a greater understanding of their taxonomy may benefit fish and human health alike. Despite focussing primarily on Canadian parasitic nematodes, these nematode species affect fishes from around the world, highlighting the value of this guide to a global readership.

WILLIAM M. TWARDEK

Fish Ecology and Conservation Physiology Laboratory, Department of Biology, Carleton University, Ottawa, ON, Canada 